\section{Antikörper effektiv gegen Kopf- und Halstumoren}

Eine Radioimmuntherapie ist bei lokal fortgeschrittenen Kopf-Hals-Tumoren bei guter Verträglichkeit vergleichbar wirksam wie eine Radiochemotherapie (RCT). Darauf weisen Daten einer im Rahmen des diesjährigen Kongresses der Deutschen Gesellschaft für Radioonkologie e.V. (DEGRO) vorgestellten quantitativen Analyse hin [Levy A. R. et al. Curr Med Res Opin. 2011;27(12): 1-7]. Sie umfasste vier Studien und eine Meta-Analyse, in denen die Wirksamkeit einer RCT mit der einer Strahlentherapie verglichen wurde. Die hieraus berechneten Hazard-Ratios [HR] für lokoregionäre Tumorkontrolle und Gesamtüberleben bei RCT wurden anschließend mit den in einer anderen Studie für die Kombination aus Cetuximab (Erbitux ${ }^{\circledast}$ ) und Radiatio ermittelten Werten [Bonner JA et al. N Engl J Med. 2006;354(6):567-78] verglichen. „Hierbei ergab sich in beiden Parametern eine vergleichbare Wirksamkeit, sodass die geringere Toxizität der Radioimmuntherapie ein wesentlicher Faktor für die weitere Therapieentscheidung werden kann“, erläuterte Marc Münter, Stuttgart.

Bei lokal fortgeschrittenen KopfHals-Tumoren ist der gegenwärtige nicht chirurgische Standard zur Behandlung die Radiochemotherapie und die Kombination von Cetuximab mit einer Radiatio. Basis dafür ist eine Phase-III-Studie [ Bonner JA et al. N Engl J Med. 2006;354:567-78], in der eine Radiotherapie mit einer Radiotherapie plus Cetuximab bei 424 Patienten mit lokal fortgeschrittenen Kopf-HalsTumoren verglichen wurde. Danach verlängerte die kombinierte Therapie die mediane lokale Tumorkontrolle gegenüber der reinen Bestrahlung $(24,4$ vs. 14,9 Monate; HR 0,68, p = 0,005) und ermöglichte ein signifikant längeres medianes Gesamtüberleben (49,0 vs. 29,3 Monate, HR 0,73; $\mathrm{p}=0,018$ ). Fünf Jahre nach Randomisierung waren noch 45,6\% der Patienten des Versuchsarms und 36,4\% der Kontrollgruppe am Leben.

Nach Informationen von Merck Serono

Zulassung

\title{
Antiangiogenese beim Ovarialkarzinom
}

\author{
Seit kurzem steht die erste antiangiogenetisch \\ wirkende Substanz für die Primärbehandlung des \\ fortgeschrittenen Ovarialkarzinoms zur Verfügung.
}

Die Zulassung von Bevacizumab (Avastin ${ }^{\circledR}$ ) basiert auf zwei randomisierten Phase-IIIStudien mit insgesamt über 3.400 Patientinnen. In der GOG-0218-Studie [Burger RA et al. N Engl J Med. 2011;365(26):247383] blieben die Patientinnen (FIGO IIIB/C, IV) unter der Bevacizumab-Gabe (15 mg/ kg KG, q3w) zusätzlich zu Carboplatin/Paclitaxel mit nachfolgender BevacizumabErhaltungstherapie über ein halbes Jahr länger ohne erneutes Tumorwachstum. Der durch Bevacizumab induzierte Vorteil entsprach einer Risikoreduktion um 35\% und war im Vergleich zum Kontrollarm ohne Bevacizumab statistisch signifikant (HR 0,644; $\mathrm{p}<0,0001$ ).

In der europäischen Phase-III-Studie ICON-7 [u.a. Perren TJ et al. N Engl J
Med. 2011;365(26):2484-96] verlängerte die Addition von Bevacizumab zur Standard-Chemotherapie mit nachfolgender antiangiogenetischer Erhaltungstherapie das progressionsfreie Überleben statistisch signifikant ( $\mathrm{HR} 0,86 ; \mathrm{p}=0,0185$ ). Im Unterschied zur GOG-Studie wurden in ICON-7 auch Patientinnen mit früheren Tumorstadien (FIGO-Stadien I/IIA, IIB-IV) eingeschlossen. In beiden Studien erhöhte Bevacizumab die Nebenwirkungsrate nicht klinisch relevant.

Birgit-Kristin Pohlmann

Pressekonferenz: „Avastin ${ }^{\circledast}$ : Zulassung beim Ovarialkarzinom. Angiogenesehemmung als neue Therapieoption" am 16. Februar 2012 in Düsseldorf; Veranstalter: Roche.

Strahlentherapie

\section{Radiochirurgie bald auch beim Prostatakarzinom?}

\section{Radiochirurgie und Tomotherapie, jeweils unterstützt durch bildgebende Verfahren, gehören zu den wirksamsten Formen moderner Strahlenbehandlung.}

Maximale Tumorkontrolle bei minimalen Nebenwirkungen - diesem Behandlungsziel lässt sich im Fall von lokal begrenzten Prostatatumoren mit stereotaktischer Radiochirurgie näher kommen, berichtete Nick van As, London, Großbritannien, im Mai in Barcelona. Die ermutigenden Ergebnisse haben zum Start der randomisierten Vergleichsstudie PACE (Prostate Advances in Comparative Evidence) geführt, in der die CyberKnife ${ }^{\circledast}$-Anwendung bei Patienten mit lokal begrenztem Prostatakarzinom mit etablierten Interventionsmethoden verglichen wird.

Laut Eric Lartigau, Lille, Niederlande, eignet sich die Radiochirurgie bald auch, um Frühstadien des Prostatakarzinoms innerhalb der Vorsteherdrüse zu behandeln. Die CyberKnife-Technologie kom- biniert ein Bildortungssystem zur kontinuierlichen Positionskontrolle mit einem kompakten Linearbeschleuniger, sodass Tumorbewegungen in Echtzeit verfolgt und korrigiert werden können.

Davon profitieren heute schon Patienten mit Lungentumoren, wie Mischa Hoogeman, Rotterdam, Niederlande, berichtete. Die Behandlung von 700 Patienten mit T1- und T2-Tumoren eines nicht kleinzelligen Bronchialkarzinoms (NSCLC) führte nach im Median zweijähriger Nachbeobachtung zu einer 96\%igen Rezidivfreiheit.

Martin Roos

Pressekonferenz und Präsentationen am 10. Mai 2012 in Barcelona anlässlich der 31. Jahrestagung der European Society for Radiotherapy \& Oncology (ESTR0-31); Veranstalter: Accuray 